\title{
Weiterer Beitrag zur pathologischen Anatomie der Skleritis.
}

Von

\author{
Prof. W. Uhthoff \\ in Breslau.
}

Hierzu Taf. XIII-XV, Fig. 1-5.

(Aus der Universitäts-Augenklinik zu Breslau.)

Meine ersten Mittheilungen zur pathologischen Anatomie der Skleritis und Episkleritis konnte ich (14) im Jahre 1883 machen. Die Untersuchungen bezogen sich allerdings nur auf excidirte Stücke des conjunctivalen und episkeralen Gewebes bei frischen Krankheitsfällen von buckelförmiger Skleritis resp. Episkleritis, so dass ich über die Veränderungen der Sklera selbst keine Angaben machen konnte. Zu jener Zeit war die einschlägige Literatur über diesen Gegenstand noch ausserordentlich gering.

Der Fall von Pilz(11) 1852 musste in seiner Deutung als eigentliche Skleritis (vielleicht Tuberculose?) zweifelhaft erscheinen, und ebenso handelte es sich in dem Falle von Noyes (10) 1873 nicht um Skleritis im gewöhnlichen klinischen Sinne. In dritter Linie kam die Untersuchung von Baumgarten (3) 1876, welche einen Fall von sklerosirender Keratitis mit starker Verdickung und entzïndlicher Infiltration der Sklera betraf, in Betracht und zuletzt eine kurze anatomische Beschreibung von sog. „purulenter Skleritis" von Alt (1) 1879 in seinem Compendium der normalen und pathologischen Histologie des Auges. 
Seit jener Zeit nun hat dieses Capitel eine Reihe von weiteren Beiträgen aufzuweisen, welche die Kenntniss in Betreff der pathologischen Anatomie der Skleritis zu fördern geeignet waren. Es sind das die Mittheilungen von Kostenitsch (9) 1894, Schirmer (12) 1895, Schlodtmann (13) 1897, Friedland (6) 1899, welche sichere Fälle von Skleritis betreffen, auch hatte ich selbst (15) 1896 noch Gelegenheit einen sicheren Fall von alter Skleritis anatomisch zu untersuchen und über denselben kurz zu berichten.

Beziehungen zu unserem Thema haben ferner die Mittheilungen ron Brailey (2) 1889, Donald Gunn(5) 1894, sowie von Gayet (7 u. 8) 1886 und 1888 und H. Coppez(4) 1895; ich werde später auch auf diese Arbeiten noch zurückzukommen haben.

Es ist nicht zu verkennen, dass in erster Linie durch die verdienstlichen Mittheilungen von Schlodtmann aus der Fuchs'schen Klinik in Wien neue Gesichtspunkte in Bezug auf' die pathologische Anatomie der Skleritis beigebracht wurden, indem er unter dem Namen der „sulzigen Infiltration der Conjunctiva und Sklera"s eine eigenartige schwere Form der Skleritis resp. Episkleritis sowohl anatomisch (erster Fall) als auch klinisch (mehrere Fälle) beschrieb und sie von dem typischen klinischen Bilde der Skleritis abzweigte. Auch der zweite Fall Friedland's (ebenfalls aus der Fuchs'schen Klinik) deckt sich mit der Beschreibung von Schlodtmann.

Ich bin nun in der Lage gewesen meine pathologischanatomischen Untersuchungen iiber Skleritis durch eine weitere Beobachtung zu vervollständigen. Der betreffende Kranke wird jetzt seit zwei Jahren von mir beobachtet, vor ungefähr Jahresfrist musste das eine völlig erblindete und degenerirte Auge entfernt werden, auch auf dem zweiten Auge ist allmäblich complete Amaurose eingetreten. Ich stehe nicht an, diesen Fall den von Schlodtmann und Friedland aus der Fuchs"schen Klinik beschriebenen 
an die Seite zu stellen. Gerade sowohl wegen der langen und genauen klinischen Beobachtung, als wegen der eingehenden anatomischen Untersuchung des einen Auges dürfte der Fall geeignet erscheinen, zur weiteren Aufklärung des fraglichen Krankheitsbildes beizutragen.

Die Krankengesehichte ist folgende: Der 75jährige Tuchmacher M. D. wurde am 29. XII. 1897 wegen einer langdauernden schweren Erkrankung seiner beiden Augen in die Universitäts-Augenklinik aufgenommen, nachdem er schon einige Wochen zuvor polikliniseh behandelt wurde.

Anamnestisch will Patient in seiner Jugend an Ausschlag am Kopf und den Extremitäten gelitten haben, vor einem Jahr litt er an Blinddarmentzündung und wurde deswegen längere Zeit auf der hiesigen Medicinischen Klinik behandelt, auch sollen die Augen schon zu jener Zeit entzündet gewesen sein, in letzter Zeit litt er sodann noch an Luftröhrenkatarrh. Specifische Infection wird in Abrede gestellt, ebenso sollen schwere rheumatische Affectionen nicht bestanden haben.

Von den Kindern des Patienten sind drei im jugendlichen Alter gestorben, während die anderen gesund sind und ebenfalls gesunde Kinder haben. Der Vater des Kranken starb an "Wassersucht", die Mutter in Folge eines Unglüekfalles, von den Geschwistern sind acht gestorben, einige ebenfalls an "Wassersucht".

Schon seit dem Jahre 1896 sollen die Augen sehr viel entzündet gewesen sein, wechselnde Intensität des Processes, auch zum Theil heerdförmiges Auftreten, so dass von ärztlicber Seite zeitweise Calomel eingestäubt wurde, also wohl an einen phlyctänulären Process gedacht wurde. Doch thaten diese Einstäubungen dem Kranken nicht gut.

Mit Anfang December 1897 nun kam der Kranke zum ersten Mal in der Universitäts-Augenklinik zu Breslau in poliklinische Behandlung und wurde am 29. XII. 1897 in die Klinik aufgenommen.

Es handelte sich um einen ziemlich sehwächlichen Mann in schlechtem Ernährungszustande, Zeichen chronischer Bronchitis, aber sonst bis auf die Augen im Wesentlichen gesund. Auch der Urin ist frei von pathologisehen Bestandtheilen.

Beide Augen boten nun das Bild einer ausgedehnten Skleritis der ganzen vorderen Bulbusabschnitte bis zum Aequator hin. An einzelnen Stellen und im Verlauf der langen Beobachtung wechselnd und wandernd trat skleritisehe Buckelbildung 
ausgesprochen zu Tage, jedoch immer so, dass auch die dazwisehenliegenden vorderen Skleralparthieen in ganzem Umfange pathologiseh affieirt blieben. Die Conjunetiva bulbi und die skleralen wie episkleralen Parthieen verdickt, von tief bläulich-röthlicher Färbung und bei der theilweisen buckelförmigen Vortreibung der erkrankten Parthieen in Verbindung mit dem langwierigen schwankenden Verlauf musste die Diagnose auf doppelseitige selr" ausgedehnte tiefgreifende Skleritis des ganzen vorderen Bulbusabsehnittes gestellt werden, mit Betheiligung der Iris (hintere Synechieen), und starken Glaskörpertrübungen. Das Sehen wurde trotz aller angewendeten Mittel immer schlechter und das linke. Ange erblindete sehon gegen Fnde Januar 1898 fast vollständig. Die Hornhaut war beiderseits in ihren Randtheilen ausgesprochen mitbetheiligt, unter dem Bilde der tiefen granen Trübung durch die ganzen Schichten der Cornea. Wegen der starken Glaskörpertrübungen kein rother Reflex mehr aus dem Augenhintergrund. Rechts noch Handbewegungen in nächster Nähe, links nur unsieberer Lichtschein.

Ende Januar 1898 verliess Patient auf seinen Wunsch die Klinik, um erst 14. III. 1898 wiederzukehren. Der Znstand war auf dem rechten Auge ziemlich derselbe geblieben, nur war die Hornhaut noch stärker in Mitleidensehaft gezogen tnd Patient auch hier bis auf unsicheren Lichtschein erblindet. Auch die Veränderungen der Sklera hatten im Wesentlichen ihren Charakter beibehalten.

Das linke Auge war inzwischen stark degenerirt. Die Ciliargegend nach aussen vom Limbus corneae war ausgesprochen ektatiseh geworden, Tens. +1 , vordere Kammer aufgehoben, namentlich in den äusseren Theilen. Der ganze vordere Bullusabschnitt zeigt noch jenes tief bräunlich-rothe Anssehen, zum Theil mit einem Stich in's gelblichgrane, bis zum Aequator hin in ziemlich gleichmässiger Weise, hochgradige skleritische Degeneration mit starker Verdünnung der nach aussen gelegenen Skleralparthieen, welche einen schiefrigen Farbenton den übrigen gegenüber bekommen haben. Die Cornea ist in den äusseren Theilen intensiv schmutaig grau, auch in ihren tieferen Schichten getrübt. Die Randparthieen der Cornea sind in den ausseren Theilen nocl etwas prominent und verdickt. Patient hat heftige Schmerzen im Auge und in der Umgebung des Auges. Da das Auge vollständig erblindet ist, wird es enucleirt und ist der Heilverlanf der Wunde ein normaler.

Nach circa $1 / 2$ Jahr am 5. XII. 1898 stell Patient sich 
mit seinem rechten Auge wieder vor, der Zustand ist ziemlich unverändert geblieben, nur ist die Cornea noch viel mehr getrübt und schmutzig grau undurehsichtig in ihren ganzen Randtheilen, so dass nur nock das Centrum etwas durchsichtig erscheint. $\mathrm{S}=0$ Lichtschein nur unsicher nach innen.

Die letzte Vorstellung des Kranken erfolgte am 21. VIII. 1899 und bot folgendes Bild des rechten Auges, im Ganzen wenig verändert gegen früher:

Der ganze vordere Bulbusabschnitt bis zum Aequator hin, soweit derselbe auch beim starken Abziehen der Lider sichtbar, gleicht einer tief bräunlich-röthlichen Aleischigen Halbkugel, welche nach aussen hin noch einen deutlich prominenten skleritischen Buckel zeigt. Nach oben am Limbus corneae hat eine $1 \mathrm{qcm}$ grosse Stelle der erkrankten Sklera etwas schmutzig gelbbräunliches in ihrem Farbenton. Sonst ist die Veränderung im ganzen vorderen Bulbusabschnitt ziemlich gleichmässig und hat eine deutliche Verdickung der ganzen Conjunctiva bulbi, der Sklera und des episkleralen Gewebes herbeigefiuhrt. Die Conjunctiva bulbi nicht verschieblich gegen die Sklera. Der Umfang des vorderen Bulbusabschnittes ist in seimen Dimensionen, eben wegen der gleichmässigen skleritischen Verdickung etwas grösser als der eines normalen Anges, jedoch bestehen hier nirgends partielle staphylomatöse Ektasieen.

Die Cornea ist intensiv sehmutzig-grau, zum Theil mit einem Stịch in's röthliche, getrübt und vollständig undurchsiehtig, jedoch besteht nirgends ein Ulcus oder eine Leukombildung nach etwa früher perforirtem Ulcus. Die Trübung der Cornea durchsetzt alle Sehichten derselben, namentlich auch die tieferen und lässt vielfach mit der Loupe eine ganz fein punktirte Zusammensetzung sowie tiefe Gefässneubildung erkennen. Von der Iris and der vorderen Kammer ist wegen dieser tiefen parenchymatösen, schmutzig-grauen Trübung der Hornhaut nielıts zu erkennen. Die Randparthieen der Cornea sind am stärksten getrübt und vaseularisirt und ist dadurch gleichsam eine scheinbare Verkleinerung der Hornhaut wenigstens im verticalen Meridian zu Stande gekommen. Der Hornhautdurchmesser beträgt in dieser Riehtung $9 \mathrm{~mm}$, in horizontaler Richtung $11 \mathrm{~mm}$. Die Randparthieen prominiren ansgesprochen gegen das Niveau der übrigen Hornhaut.

Bei Loupenbetrachtung erkennt man nun, wie im ganzen vorderen Bulbusabsebnitt jo Bereich der erkrankten Sklera die oberflächliche Schicht bis zu einem gewissen Grade homogen 
durehscheinend ist, so dass man die vermehrten und ektatischen Gefässe der episkleralen Parthieen durchscheinen sieht.

Namentlich sind es die Verästelungen der vorderen Ciliargefässe, welche in der Tiefe dieser homogenen bräunlich graulichen Schicht blass roth durchscheinen. Dieselben sind enorm erweitert und geschlängeit und scheinen vielfach, wie mit der Loupe zu erkennen, etwas weissliche getrübte verdickte Wandungen zu haben. An einzelnen Stellen erkennt man in den sulzig infiltrirten oberflächlichen conjunctivalen und episkleralen Schichten kleine blass grau durchscheinende, flache, knötchenförmige Bildungen. In einem anderen Theil der Sklera z. B. nack aussen ist ein stärkerer skleritischer Heerd noch sichtbar; der auch eine intensivere, dunkel-bräuniich-violette Färbung zeigt, als die übrigen Parthieen. Das Auge ist relativ gut beweglich, Tension - 1,0, keine Schmerzhaftigkeit bei Berïlrung. Dieses Aussehen mit allmählich etwas zunehmender Intensität der Veränderungen bietet das rechte Ange jetzt somit seit zwei Jahren, und ganz analog war auch der Process seiner Zeit añf dem enucleirten linken Ange, dessen anatomische Beschreibung jetzt folgen soll.

Das Allgemeinbefinden des Kranken ist bis in die letzte Zeit relativ gut geblieben, er ist jetzt 77 Jahre und zeigt, abgesehen von seiner alten Bronchitis, keine sonstigen hervorstechenden Krankheitserseheinungen, besonders auch keine Erscheinungen Fon Gicht und Rheumatismus.

Anatomische Beschreibung: Der linke enucleirte Bulbus wurde frish in Muller-Formol gehärtet und in dankenswerthester Weise von Herra Dr. Seydel in Celloidin eingebettet und gesehnitten. Färbung mit Haematoxylin, Alauncarmin, Doppelfärbung mit Haematoxylin und Eosin nach van Gieson, Bakterienfärbung nach Weigert u. s. w.

Wie ein horizontaler Durehsehnitt durch den Augapfel zeigt (siehe Fig. 1), ist der ganze rordere Bulbusabschnitt ungefähr bis zum Aequator nach hinten reichend der Sitz hochgradiger Veränderungen. Es besteht hier, gürtelförmig die Hornhaut umgebend, eine mächtige entzïndliche Infiltration der ganzen Bulbuswanduagen mit starker Verdiekung derselben. Dieselbe beträgt etwas hinter der Ciliargegend an Stelle der stärksten Veränderung, nach aussen eirea $3,5 \mathrm{~mm}$, nach innen bis $4,5 \mathrm{~mm}$. Die Breite dieser erkrankten Zone beträgt eirca 9-10 $\mathrm{mm}$ in der Richtung von vom nach hinten. Die stark entzindlich infiltrirte und rerdiekte Conjunctiva bulbi ist fest mit der darunter liegenden 
und ebenfalls infiltrirten und zum Theil völlig dilacerirten Sklera, sowie weiter zusammenhängend mit der darunterliegenden mächtig gewucherten und verdickten infiltrirten Chorioidea verwachsen, so dass die Bulbushüllen fest zusammenhängend jene oben erwähnten Dickenmaasse erreichen. Die Schnittserien zeigen nun, wie diese entzündliche, dichte, zellige Infiltration mit starker Verdickung und Verwachsung der Bulbushüllen den ganzen vorderen Bulbusabschnitt ziemlich gleichmässig gürtelförmig bis zum Aequator occupirt. Die Cornea ist nur in ihren Randtheilen, namentlich von aussen her mit ergriffen.

Anf der änsseren Oberfläche des Angapfels hört die Veränderung am Aequator durchweg auf, nur vereinzelte heerdförmige entzündliche Infiltrationen in der Umgebung der Gefässe erstrecken sich auch noch weiter nach hinten und reichen vereinzelt bis an den hinteren Bulbuspol (siehe Fig. 1), während sonst die Verhältnisse hier normal sind. Man sieht jedenfalls wie die eigentlichen Propagationsbahnen des Processes weiter nach linten lediglich die Umgebrng der Gefässe sind, es zeigt sich das besonders significant auch da, wo ein Gefäss auf dem Längsschnitt getroffen ist, welches dann mit einem scheidenförmigen Zellmantel versehen ist.

Die Affection nun in dem vorderen Bulbusabschnitt erweist sich anatomisch als hochgradige Episkleritis, Skleritis und Chorioiditis. Das infiltrirende zellige Material sind durchweg einkernige Leukoeyten, welche sowohl die Conjunctiva bulbi, als die episkleralen Theile, die Sklera selbst und die entsprechenden Theile der Chorioidea dicht durchsetzen.

Die Sklera ist in einer ringförmigen Zone, parallel zum Limbus corneae hochgradig infiltrirt, ja zum Theil direct durchbrochen und zerstört. Die entzündliche Infiltration erstreckt sich streifenförmig zwischen die aufgelockerten Skleralfaserschichten hin. An einzelnen Stellen ist von Skleralgewebe nichts. melr zu seben und hier sieht man an der Grenze der Durchbruchsstellen, wie die Skleralfasern sich pinselförmig anseinanderbreiten, sich in feinere Fibrillen auflösen und schliesslich völlig zu Grunde gehen. Auch die noch vorhandenen Sklerallagen in dieser Gegend zeigen zum Theil Degeneration, welche sich in mangelńder Färbbarkeit der Kerne der betreffenden Parthieen documentirt. Besonders hervorzuheben ist noch für die Stellen, wo die Sklera durchbrochen und wo die Skleralsehichten sich pinselförmig auflösen, ein ausgesprochener Zerfall der Zellkerne. Die Fragmentation der Zellkerne (Chromatolyse) ist an diesen Stellen viel- 
fach ausserordentlich hervortretend, während sie in den ubrigen Theilen des erkrankten Terrains, bis auf einige Stellen in der Chorioidea sich nicht findet. Im hinteren Abschnnitt des Bulbus ist die Sklera völlig intact erhalten und zeigt hier auch gat gefärbte Zelikerne. Nach aussen vom Limbus corneae ist die Sklera, abgesehen von ihrer starken entzündlichen Infiltration ausgesprochen ektatiseh (siehe Fig. 1). Diese Ektasie hatte sick intra vitam unter Drucksteigerung ziemlich sehnell entwickelt und reprësentirte sich als partielles Intercalarstaphylom mit dunkler Verfärbung nach aussen vom Limbus.

Auf allen Durchschnitten zeigt sich, dass die grösste Intensität des perforirenden skleritischen Processes nicht unmittelbar am Limbus ihren Sitz hat, sondern eirea $2-3 \mathrm{~mm}$ davon entfernt in der Gegend, wo die vorderen Ciliargefässe die Sklera perforiren. Von diesem Terrain aus erstreekt sich dann der Process, gleichsam "manschettenknopfartig:", sowohl in das äussere episklerale und subconjunctivale Terrain, als anch nach innen, sich in der Chorioidea ausbreitend (siehe Fig. 1 und 2); an der Durchbruchstelle zeigt die Aderlant die stärkste entzündliche Infiltration, welche von hier aus nach rorn sowohl (Corpus ciliare), als nach hinten almählich ablklingt. Die starke episkleritische und subconjunctivale entzundliche Infiltration reicht nach rorn bis in den Limbus hinein und bildet eine wallförmige $\mathrm{Er}$ hebung rings um die Cornea, anf der äusseren Seite erstreckt sie sich cirea $1,5 \mathrm{~mm}$ in die oberen und mittleren Cornealschichten hinein in Form von längsstreifigen Zellzügen. Nach binten zo von den stärkst afficirten Skleralstellen reicht der Process in den oberflächlich skleralen, episkleralen und subconjunctivalen Theilen anch noch circa $3-4 \mathrm{~mm}$ rückwärts, allmählich an Intensität abklingend und sich in jenen oben beschriebenen vereinzelten perivaseulitischen Zellzügen, welche selbst gelegentlich bis an die Gegend des linteren Bulbuspoles reichen, verlierend.

In diesen mächtigen wallförmigen skleralen, episkleralen und subconjunctivalen Veränderungen sind neben der entzündlichen Zellinfiltration mit einkernigen Leukoeyten, die Gefässveränderungen die hervorstechendsten Erscheinungen, Dieselben sind stark erweitert und gewuchert, sodass das Gewebe z. Th, ein völlig cavernöses Aussehen dabbietet (s. Fig. 2). Es ist stellenweise gar nicht zu verkennen, dass der Aufbau der entzündlichen Infiltration vielfach gerade durch das Verhalten der Gefässe gezeben wurde and dadurch ein netzförmiges Aussehen gewinnt. Die Gefässe 'auf dem Quersehnitt enthalten vielfach lediglich 
Blut, indem die rothen Blutkörper dicht gedrängt an einander liegen und das Gefäss ganz ausfüllen. Auf andern Querschnitten ist nur ein Theil des Querschnittes. durch einen Haufen rother Blutkörper eingenommen, während der übrige Theil von homogener geronnener Flüssigkeit erfüllt ist. Ein Theil der Blutgefässquerschnitte zeigt gar keine geformten Blatelemente, sondern lediglich homogenen Inhalt. Es zeigt sich jedenfalls in diesem Verhalten, dass intra vitam eine sebr starke Circulationsstörung in den Gefässen vorhanden gewesen sein muss, ja z. Th. ist es gerechtfertigt von hyaliner Thrombose zu sprechen.

Die Wandung dieser stark erweiterten und vermehrten Gefässe ist durchweg sehr dünn, das auskleidende Endothel gewöhnlich gut zu erkennen. An andern Gefässen ist deutliche Sklerose der Wandungen erkennbar, und ebenso Endothelwucherung mit Verlegung oder starker Verengung des Lumens. An einzelnen Stellen finden sich Blutungen in dem entzündlich infiltrirten Gewebe, an andern geformte homogene und feinfibrilläre Exsudatmassen. Ausser den Blutgefässdurchschnitten handelt es sich namentlich in den oberfläehlichen subepithelialen Conjunetirallagen um erweiterte und vermehrte Lymphgefässe. Die Lumina derselben sind vielfach ganz leer, zum Theil zeigen sie einen homogenen ganz gleichmässigen Inhalt. Ein Endothelbelag ist durchweg auf den Querschnitten nachweissbar, eine eigentliche wohlausgeprägte Wandung ist nicht wahrzunehmen.

Diese Veränderungen des Blut- und Lymphgefässsystems deeken sich im Ganzen mit den in meinen frühern Fällen gefundenen und beschriebenen. Ich glanbe, dass gerade ihnen eine grosse Rolle für den Aufbau der skleritisehen und episkleritischen Processe zukommt.

Neben diesen bisher beschriebenen Veränderungen der entzündlichen Infiltration, den Gefässveränderungen u. s. w. finden sich ferner mehr oder weniger ausgesprochen die Zeichen von Gewebsproliferation, welche ror allem in der theilweisen Vermehrung der grossen länglich ovalen Zellkerne ihren Ausdruck findet. Es ist in dieser Hinsicht noch besonders hervorzuheben das Vorhandensein ziemlich zahlreicher kleiner knötchenförmiger Bildungen, die meistens in den oberflächlichen subconjunctivalen, z. Th. aber auch in den tiefern episkleralen Parthieen sich finden (s. Fig. 1, 3 und 4). Bei genauerer und namentlich bei Loupenbetrachtung waren dieselben schon makroskopiseh am lebenden als kleine grauröthliche Knötchen sichtbar. Ihre Grösse wechselt erheblich, jedoch können sie einen Durchmesser bis zu 
$1 \mathrm{~mm}$ erreichen. Mikroskopisch zeigen dieselben ziemlich constant sich aufgebaut aus zweierlei Zallmaterial. Der Kern, der sich deutlich von dem umgebenden Mantel differenzirt, besteht aus endothelialen Zellen mit grössern blass gefärbten, oft feingranulirten, ovalen und rundlichen Kernen mit deutlichen Kernkörper. chen. Ein kleiner Theil dieser Zellkerne färbt sich kaum noch mit Haematoxylin und zeigt gelegentlich ein mehr blasenartiges, homogenes, klumpiges Aussehen, wobei das glänzende Kernkörperchen gewöhnlich noch sichtbar ist, es sind dies wobl zweifellos Zeichen der Degeneration. Durchweg aber hat man es jedenfalls mit lebendem Zellmaterial zu thun, ja zum Theil sind Kerntheilungsfiguren als Zeichen von Proliferationsvorgängen nachweisbar, jedenfalls fehlen Verkäsung; ansgedehntere Nekrose, Riesenzellen völlig in diesen centralen Parthieen der Knötchen (s. Fig. 3 u. 4).

Die umgebende mantelartige Zone dieser centralen Knötchenparthieen besteht gleichmässig aus den einkernigen infiltrirenden Lenkocyten, deren Kerne sehr intensiv tingirt ist. Die Grenze zwischen diesem peripheren Infiltrationsring und dem andersartigen Kern des Knötchens ist oft ganz seharf ausgesprochen, zum Theil aber erkennt man anch mikroskopisel, wie theilweise in disseminirter Weise einzelne einkernige Leakocyten zwischen die central liegenden endothelialen Zellen eingewandert sind (siehe Fig. 4).

In einer Anzahl dieser Knötchen ist es möglich, ein spärliches capillares Gefässnetz nachzuweisen, welches sowohl den peripheren Mantel als den Keru des Knötchens durchsetzen kann, gewöhnlich dann aber in den Kernparthieen deutlicher wahrnehmbar ist (siehe Fig. 4).

Trotz vielfacher Bemuihungen ist es mir nicht mit Sicherheit gelungen, die Genese und die Bedeutung dieser Knötchen genauer festznstellen. Für einen kleinen Theil dîxte folgender Entstehungsmodus zutreffend sein, wie ich an der hintern Grenze des skleralen und episkleralen Processes gelegentlich naehweisen zu können glaube (siehe Fig. 3). Man sieht hier zuweilen, wie ein infiltrirender Zellmantel einkerniger Leukocyten ein kleines Gefässlumen mit gewucherten Endothelien und vollständig, verlegtem Lumen umgiebt, und wie dann auf diese Weise das Bird ganz kleiner knötchenförmiger Heerde mit einem Centrum von endothelialen Zellen und umgebender Infiltrationszone einkorniger Leukocyten zu Stande kornmt. $\mathrm{Ob}$ in dieser Weise etwa unter Wucherung des central gelegenen endothelialen Zellmaterials und völligem Zngrundegehen der dinnnen Gefässwandung auch die 
grössern Knötchen zu Stande kommen, möchte ich dahin gestellt sein lassen, jedenfalls konnte dieser Nachweis an den grösseren Knötchen nicht direct geführt werden.

Ganz vereinzelt wurde ein derartig zusammengesetztes Knötchen auch in der Tiefe des Auges in der stark verdickten Chorioidea angetroffen.

Die Cornea war bei diesem anatomisch untersuchten Auge noch relativ intact geblieben, nur in die Randtheile derselben und namentlich anf der äussern Seite war die entzündliche Infiltration in Form von Zellzügen in die oberflächlichen und mittleren Hornhautschichten übergegangen. Sonst Epithel und Bowmansche Membran durchweg gut erhalten, ebenso das Hormhautparenehym und die Membrana Descemetii mit ihren Endothelbelag; auf letzteren fanden sich mehrfache Sehichten einkerniger Lenkoeyten aus dem vordern Kammerwasser niedergeschlagen.

Die Epithelschicht der Conjunctiva im Bereich des erkrankten Terrain's ist continuirlich erhalten nod erseheint in grosser Ausdehnung verdickt, namentlich in der Nähe des Limbus auch zapfenförmige Fortsätze in die Tiefe sendend. An einzelnen Stellen sieht man scheinbar isolirte Nester von Epithelzellen abgetrennt von der obern Epithelschicht im erkrankten Conjunctivalgewebe, es sind das derartige quergetroffene Epithelzapfen, die nur auf dem Schnitt isolirt in der Conjunctiva zu liegen scheinen, sonst aber continuirlich mit der Epithelschicht im Zusammenhang stehen.

Der Uvealtractus zeigt sehr hochgradige Veränderungen, jedoch lässt sich an der ganzen Topographie auf den Schnittserien erkennen, dass der Process offenbar von den stärkst erkrankten sklexitischen Parthieen, namentlich da, wo es zur Zerstörung und zum Durchbruch der Sklera gekommen ist, seinen Ausgangspunkt genommen hat. Einmal aber in das Augeninnere übergegangen, haben die chronisch entzündlichen Veränderungen der Chorioidea mächtig sowohl nach vorn als nach hinten übergegriffen. Nach hinten erstreckt sich die entzïndliche Infiltration und Verdickung der Chorioidea mit stetig abnehmender Intensität bis an die Gegend des Sehnerven-Eintritts; nach vorn ist das Corp. ciliare sehr stark in Mitleidenschaft gezogen; so dass von der Structur desselben kaum noch etwas zu erkennen. Dieser Theil des Uvealtractus ist ebenfalls in stark zellig infiltrirte, verdickte, entzündliche Masse umgewandelt. Die Ciliarfortsätze sind nicht zu erkennen and sicher auch functionell ganz zu Grunde gegangen. Auch die Iris ist noch stark in Mitleidenschaft gezogen, 
im äussern staphylomatösen Theil liegt sie völlig der Hinterfläche der Cornea an, so dass die rordere Kammer hier ganz anfgehoben ist. Nach innen $z \mathrm{n}$ ist sie mit der Linse verwachsen. Die Iris zeigt einerseits entzündliche Infiltration, andererseits ausgesprochene Atrophie.

Die entzündlichen Veränderungen der eigentlichen Chorioidea sind sehr hochgradig, und erreicht die Membran stellenweise eine Dicke bis zu $3 \mathrm{~mm}$. Auf der äussern temporalen Seite nimmt die entzündliche Verdickung der Chorioidea nach hinten von der skleritischen Perforation an continuirlich ab, sodass in der Gegend des Opticuseintritts nur noch eine geringfügige zellige Infiltration vorhanden ist. Sie bietet im Ganzen das Bild einer" hyperplastischen Chorioiditis, ähnlich wie man es so häufig in cyclitischen Augen findet, die zu sympathischer Entzïndung Veranlassung gegeben haben. In den vordern, am stärksten betroffenen Parthicen sind die Chorioidealgefässe eigentlich völlig zu Grunde gangen, im hintern Abschnitt sielt man noch zahlreiche relativ normal grosse Gefässquersehnitte, doch sind auch gerade hier Endarteriitis, Perivasculitis, Sklerose der Gefässwandungen, Thrombose, völlige Obliteration des Lumens besonders häufig anzutreffen (siehe Fig. 5).

Es ist in erster Linie die Schicht der mittelgrossen Geflisse hiervon betroffen. Die Chorioidea ist fest mit der Sklera verwachsen.

Anf der inneren Seite ist die Verdickung der Chorioidea am stärksten. Wie nach aussen besteht auch hier die stärkste entzünaliche Infiltration entspreehend den starken skleritischen Veränderungen im vordern Abschnitt, weiter nach hinten nimamt dieselbe $a b$, indem sie die äusserste und innerste Schicht vor Allem betrifft, die mittlere aber mehr frei lässt. Dafür aber findet sich hier in den mittleren Chorioidealsehichten eine mächtige Durchtränkung mit serösen und beim Härten geronnenen ExsudatMassen, ja auf eine grosse Strecke hin (cirea $6 \mathrm{~mm}$ lang und bis $\mathrm{zu} 1,5 \mathrm{~mm}$ breit) ist es zur. Bildung eines grossen eystischen Spaltraumes gekommen, der mit homogenem, mässig rundzellenhaltigem. Exsudat erfült ist. Die äussern and die inneren Chorioideallagen sind hier weit klaffend anseinander gewichen (siehe Fig. 1 und 2). Auch in der Umgebung des Spaltraums erkennt man, wie das Chorioidealstroma ron serösem Exsudat durehtränkt ist, und wie dadureh die Bestandtheile der Chorioidea (Stromapigmentzellen u. s. w.) weit auseinandergedrängt sind. Stellenweise finden sich in diesem Teritorrium ausserdem zahlreiclte 
Weiterer Beitrag zur pathologischen Anatomie der Skleritis. 551

klumpige gelbe Pigmentmassen verstrent, die wohl als ats alten Blutungen stammend anzusehen sind. Das Pigmentepithel auf der Chorioidea ist zum Theil noch erhalten. - An einzelnen Stellen der stark infiltrirten und entziundlich veränderten äussern Chorioidealschichten sind spärliche Riesenzellen nachweisbar.

Auf die ubrigen intrancularen Veränderungen will ich hier nicht näher eingehen, weil sie als secundäre anzusehen sind und für das Wesen des Processes nicht von Bedeutung.

Es besteht eine complete trichterfơrmige Netzhantablösung mit weitgehender Degeneration der Retina namentlich in ihren äussern Schichten.

Der Nervus opticus ist total atrophiseh und enthält keine gesunden Nervenfasern mehr.

Die Linse ist nach aussen and vorn luxirt and liegt mit ihren äussern Theilen der Hinterfläche der Hornhaut und der mit ihr verwachsenen Iris an. Die vordere Corticalis der Linse zeigt ausgesprochenen cataractösen Zerfall.

Der Querdurchmesser des horizontalen Bulbusdurehsehnittes beträgt $26 \mathrm{~mm}$, ebenso der Längsdurchmesser von äusserer Oberfläche zu äusserer Oberfläche gemessen.

Epikrise: Bei einer resümirenden Betrachtung unseres Falles sowohl in klinischer als anatomischer Hinsicht, möchte ich denselben den drei Fällen von Schlodtmann, von denen der erste zur anatomischen Untersuchung kam, und dem Fall II von Friedland, der ebenfalls anatomisch untersucht wurde, anreihen, und es scheint mir völlig gerechtfertigt, diese Krankheitsbilder von dem gewöhnlichen Bilde der buckelförmgen Slkleritis resp. Episkleritis als etwas Besonderes abzusondern. Freilich handelt es sich meines Erachtens auch bier in erster Linie um eine skleritische und episkleritische Affection mit secundärer Hornhautaffection, aber eine Erkrankung die durch eine Reihe besonderer Merkmale ausgezeichnet ist. Ich habe diese Beobachtung als die einzigste in ibrer Art unter meinem $\mathrm{Ma}$ terial zu verzeichnen (unter circa 100000 Augenkranken), so zahlreich auch sonst die Fälle von Skleritis resp. Episkleritis waren.

v. Graefe's Archir für Ophthalmologie. XLIX. 3. 
Es scheint zunächst, als ob diese Erkrankung in erster Linie Patienten im höheren Lebensalter befällt.

Die fünf Kranken standen im Alter von $64-75$ Jahren, vier waren über 70 Jahre alt.

Der Process verlief in meiner Beobachtung enorm chronisch unter Exacerbationen und Remissionen, ähnlich in den übrigen Fällen.

Durchweg trat die Erkrankung doppels eitig auf, wenn auch nicht immer gleichzeitig auf beiden Augen, nur in Fall III ron Schlodtmann war nur das rechte Auge befallen.

Die Prognose scheint durchweg eine sehr ernste zu sein, und im Verlauf von zwei Jahren erblindete unser Patient völlig, trotz aller aufgewandten Mittel. Aehnlich in den übrigen Fällen bei den befallenen Augen, nur in Fall I (Schlodtmann) heilte der Process auf dem zweiten in geringerem Grade befallenen Auge aus und zwar unter partieller bläulicher Verfärbung der vorderen Skleralparthieen wie nach Skleritis, während das erste wegen starker Degenerationserscheinungen mit Schmerzen und Erblindung enucleirt werden musste.

Gerade diese schwere Form der sklexitischen und episkleritischen Erkrankung hat relativ häufg zur Enucleation Veranlassung gegeben trotz ihres an und für sich seltenen Vorkommens, während die gewöhnliche buckelförmige recidivirende Skleritis nur relativ selten zur anatomischen Untersuchung kam im Verhältniss zu ihrem häufigen Auftreten.

Das Ausseben, die Ausbreitung und die Localisation des Processes in unserem Falle, wenigstens in den späteren Stadien, deckt sich weitgehend mit der Beschreibung von Schlodtmann und Friedland, jedoch war ich in der Lage innerhalb zweier Jahre den Process auch lklinisch $\mathrm{zu}$ verfolgen und auch theilweise noch die früheren Stadien der Entwicklung zu beobachten und gerade 
Weiterer Beitrag zur pathologischen Anatomie der Skleritis. 553

in dieser Hinsicht möchte ich herrorheben, wie vielfach das Bild einer wechselnden, ausgesprochenen, skleritischen Buckelbildung dabei zu Tage trat, wenn auch der Process von vornherein eine grosse Neigung zeigte sich über den ganzen vorderen Skleralabschnitt auszudehnen. In den späteren Stadien war die Bezeichnung einer mehr gleichmässigen gürtelförmigen Affection im vorderen Bulbusabschnitt vom Limbus bis zum Aequator des Bulbus durchaus gerechtfertigt und das Bild blieb an dem rechten nicht enucleirten Auge während Jahresfrist ziemlich unverändert. Der ganze vordere Bulbusabschnitt zeigte eine bräunlichröthliche Verfärbung mit völlig tiefer Trübùng der Cornea, auch ein sulziges homogenes Aussehen des vorderen Bulbusabschnittes trat in diesen späten Stadien deutlich zu Tage, wie oben geschildert, und erhielt die Verfärbung stellenweise einen ausgesprochen schmutzig bräunlichgelben Farbenton.

Die infiltrirte Limbusgegend setzte sich deutlich prominent gegen die getrübte Hornbaut ab.

So wie in den Fällen von Schlodtmann und Friedland localisirt sich der schwere skleritische und episkleritische Process ausschliesslich auf den vorderen Bulbusabschnitt und hört jenseits des Aequators nach hinten ganz auf; während die inneren Veränderungen des Auges speciell die Chorioidealveränderungen, wenn auch mit abnehmender Intensität sich weiter nach hinten erstrecken. Am ausgesprochendsten sind aber in der Chorioidea die entzündlichen Erscheinungen entsprechend den stärkst afficirten vorderen Skleralparthieen. Ich möchte deshalb für meinen Fall auch annehmen, dass die Affection der Chorioidea eine secundäre ist und ausging von der Stelle, wo der skleritische Process die Sklera durchsetzte und zum. Theil zerstörte. Die eigentliche Sklera ist ja offenbar für die Ausbreitung eines entzündlichen Processes ein ungünstiges Terrain, und so glaube ich, ist es nicht zu verwundern, wenn bei dieser 
Form einer durchschlagenden Skleritis die Sklera selbst nur relativ circumscript lädirt erscheint, während der entzündliche Process sowohl in den episkleralen und subconjunctivalen Parthieen, als auch vor Allem in der Chorioidea eine viel grössere Ausbreitung zeigt. Es liegt auch in unserem Falle, keine Veranlassung vor, die Affection der Chorioidea als eine primäre und für sich bestehende anzusehen, sondern ich bin geneigt, die inneren Veränderungen des Angapfels lediglich als secundäre zu betrachten und in einer Skleritis resp. Episkleritis den Ausgangspunkt der ganzen Affection zu sehen, namentlich auch mit Rücksicht auf die klinische Entwicklung der Erkrankung. Die Sklera selbst ist ja aber offenbar in erster Linie dort für eine entzündliche Affection disponirt, wo die vorderen Ciliargefässe (Venen sowohl wie Arterien) dieselbe durchsetzen.

Auch Friedland führt fitr seinen Fall II aus, dass die sulzige Infiltration der Episklera das Primäre war, bei guter Function des Auges, während die Erkrankung der Chorioidea, der Verfall des Behvermögens und weitere tiefere Veränderungen erst später hinzutraten.

Im vorgeschrittenen. Stadium scheint die Affection, wie in unserem Falle, ziemlich gleichmässig den ganzen vorderen Bulbusabschnitf gürtelförmig zu occupiren und vom Limbus aus sich unter dem Bilde der tiefen parenchymatösen Trübung in die Hornhaut hineinzu erstrecken. Auf dem zweiten nicht enucleirten Auge unseres Patienten führte wäbrend circa 11/2 juhrigen Beobachtungszeit der Process zu einer completen und danernden tiefen parenchymatösen Trübung mit oberflächlicher und tiefer Gefässneubildung. Die Frkrankung der Cornea ist eine secundäre durch den skleritischen Process bedingte.

In Bezug auf die Natur des Processes ist herrorzuheben, dass es sich nicht um eitrige Entzündung handelt, die infiltrirenden Zellen sind durchweg einkernige Leukocyten, wie sie dem Granulationsgewebe zukommen. 
Weiterer Beitrag zur pathologischen Anatomie der Skleritis. 555

Ausgesprochene nekrotische Heerde, wie sie von Friedland und Schlodtmann beschrieben, finden sich nicht, nur in einzelnen Parthieen der Sklera im Bereich der grössten Intensität des Processes sind die Kerne nicht mehr färbbar, als Zeichen der Degeneration. Auch die endothelialen Zellen im Centrum der runden knötchenförmigen Heerde zeigen wohl eine blassere Färbung ihrer grossen ovalen Kerne, doch ist durchweg die Färbung noch deutlich, so dass man auch hier nicht von Nekrose sprechen kann. Ja, es lassen sich, wenn auch sparsam, noch Kerntheilungsfiguren nachweisen, ein Zeichen, dass auch hier noch Proliferationsvorgänge stattfinden. An einem Theil dieser Zellen ist der Kern jedoch nicht mehr färbbar, auch die Form mehr klumpig als Zeichen der Degeneration. Zur Bildung von Riesenzellen ist es durchweg nicht gekommen, ganz vereinzelt begegnet man ihnen in den äusseren Lagen der stark verdickten Chorioidea.

Bemerkenswerth ist noch im Bereich der Skleralschichten, da wo die Auffaserung und Zerstörung derselben am intensivsten sind, ausgesprochener Kernzerfall der infiltrirenden einkernigen Leukocyten, auch in den tieferen Schichten der verdickten Chorioidea findet sich zum Theil eine derartige ausgedehnte Kernfragmentation (Chromatolyse). Es ist dies ein ausgesprochenes Zeichen der Zelldegeneration.

Veränderungen im Bereich des Gefässsystems und zwar sowohl der Blut-als der Lymphgefässe spielen als anatomische Factoren in diesem Krankheitsbilde eine ganz hervorragende Rolle. Im Bereich des vorderen Bulbusabschnittes in den erkrankten conjunctivalen und episkleritischen Parthieen besteht eine mächtige Gefässvermehrung und Erweiterung derselben, so dass das krankhaft veränderte Gewebe zum Theil ein ganz cavernöses Aussehen zeigt. In dem oberflächlichen subepithelialen Parthieen finden sich auch zahlreiche stark erweiterte mit ein- 
fachem Endothelbelag ausgekleidete Lymphräume. Dieselben sind zum Theil leer, zum Theil mit einer homogenen Masse gefüllt. An dem Inhalt der Blutgefässquerschnitte zeigt sich, dass jedenfalls intra vitam eine starke Circulationsbehinderung bis zur Thrombose einzelner Gefässe bestanden hat. Vielfach sind die stark erweiterten Gefässquerschnitte prall mit Blut gefüllt, oft aber zeigt nur ein Theil des Querschnittes rothe Blutkörper, welche in einen homogenen geronnenen Inhalt eingebettet sind.

Veränderungen der Gefässwandungen sind vielfach nachweisbar, sowohl in den vorderen eigentlich skleritischen und episkleritischen, als auch besonders in den weiter nach hinten liegenden episkleralen Parthieen, besonders aber auch in der stark veränderten Chorioidea. Vielfach ausgesprochene sklerotische Verdickung der Wandungen mit Verengerung und fast völliger Verlegung des Lumens, Endothelwucherung mit völliger Obliteration des Gefässes. Die hervorstechendste und verbreitetste Veränderung ist die entzïndliche Infiltration in der Umgebung der Gefässe. Es ist namentlich markant, wie in den binteren Theilen der erkrankten Parthieen der Process sich fast nur noch als entzürdliche Infiltration in der Umgebung der Gefässabschnitte abspielt, und selbst bis an dem hinteren Pol des Bulbus finden sich vereinzelte isolirte Gefässquerschnitte mit stark infiltrirter Scheide, während alles Uebrige normal erscheint. Aber auch in dem eigentlichen Krankheitsterrain im vorderen Bulbusabschnitt ist vielfach noch erkennbar, wie perivasculitische Veränderungen und entzündliche Vorgånge in der Ungebung der Gefüsse die Form des Processes beherrschen. Diese Veränderungen des Blutgefässsystems und der Lymphgefässe sind auch bei meinen früheren Fällen mit die hervorstechendsten Erscheinungen.

An verschiedenen Stellen finden sich auch Blutungen in dem erkrankten Gewebe, die nicht auf den operativen Eingriff zurückzuführen sind. 
Weiterer Beitrag zur pathologischen Anatomie der Skleritis. 557

Ueber die Natur der Knötchen, welche oben beschrieben, vermag ich nichts Bestimmtes auszusagen. Sie ähneln eigentlich am meisten dem Trachomfollikel. Stets war ein differentes Centrum von grösseren endothelialen Zellen mit grösseren blasser tingirten Kernen vorhanden and ein dichter Infiltrationsmantel einkerniger Leukocyten. Zum Theil ist die Abgrenzung dieser beiden Zellsorten gegeneinander eine ganz scharfe wie schon makroskopisch an den gefärbten Schnitten deutlich. Mikroskopisch sieht man jedoch, wie auch vielfach an der Grenze die einkernigen Leukocyten in disseminirter Weise in den Kern des Heerdes eindringen. An einzelnen Stellen, glaube ich, die Entstehung des Centrums dieser Knötchen aus Endothelwucherung eines kleinen Gefässquerschnittes mit völliger Obliteration desselben nachgewiesen zu haben, um welchen sich dann die entzündliche ringförmige Infiltration mit einkernigen Leukocyten bildete (s. Fig. 3). Meistentheils aber war ein solcher Entstehungsmodus nicht nachweisbar und möchte ich daher diese Erklärung nicht immer für die zutreffende halten.

Mit dem Bau des Tuberkels boten diese Gebilde keine Analogie, keine Verkäsung, keine Riesenzellen u. s. w. Auch mit Lymphfollikeln sind sie nicht zu verwechseln, wegen ihrer differenten Zusammensetzung im Centrum und in der Peripherie, mit dem Trachomfollikel existiren gewisse Analogieen, jedoch lagen sonst in keiner Weise trachomatöse Veränderungen der Bindehaut vor. Auch makroskopisch waren in der sklexitischen Zone ror der Enucleation schon diese kleinen graugelblichen Knötchen zu sehen, ebenso wie an dem zweiten nicht enucleirten Auge. Ihre Lage war zumeist eine oberfächliche, zum Theil aber auch eine tiefere in den äusseren erkrankten Skleralschichten selbst. Ich möchte glauben, dass wir es hier mit analogen Gebilden zu thun haben, wie wir sie auch gelegentlich bei dem Krankheitsbilde der gewöhnlichen buckelförmigen Skleritis und Episkleritis auftreten sehen. 
Die Untersuchung auf Mikroorganismen nach Weigert, Loeffler u. s. w. fiel negativ aus.

Aetiologisch gelingt es nicht, in unserem Falle etwas Bestimmtes nachzuweisen, eine chronische Bronchitis ist neben dem hohen Alter des Patienten die einzige complicirende Erscheinung. Von specifischer Infection nichts nachweisbar, ebenso ist eine tuberculöse Natur der Erkrankung lauszuschliessen, auch die diffuse Ausbreitung einer doppelseitigen Geschwulstbildung mit Uebergreifen auf die Bulbi wie in den Fällen von Gayet und dem einseitigen Fall von Coppez ist von der Hand zu weisen. Nach der Enucleation bleibt die Orbita völlig frei von weiteren krankhaften Veränderungen und ebenso bleibt auf dem zweiten Auge der Process constant in derselben. Weise während einer ${ }^{1}{ }_{2} \%_{2}$ jährigen Beobachtungszeit stationär. Und doch wie ähnlich ist $z$. B. die von Coppez gegebene Abbildung den anatomischen Veränderungen in unserem Falle in Bezug auf das Ergriffensein des vorderen Bulbusabschnittes.

Des Weiteren erscheint auch eine leukämische oder pseudolenkämische Affection oder ein lymphomatöser maligner Process in unserem Falle ausgeschlossen, da weder der Blut- noch Lymphdrüsenbefund sowie sonstige begleitende Erscheinungen hierfuir Anhaltspunkte ergeben.

An Schlodtmann und seinen Erwägungen mich anschliessend, glaube ich, dass auch die Fälle von Donald Gunn von syphilitischer(?), subconjunctivaler Infiltration des vorderen Bulbusabschnittes und den Fall von Brailey, der wohl zweifellos als Tuberculose der Conjunctiva bulbi et palpebrarum aufzufassen ist, nicht hierher zu rechnen sind.

Alles in Allem möchte ich den Krankheitsprocess in unserem Falle als eine besondere Form schwerer durchschlagender, chronischer, doppelseitiger Skleritis und Episkleritis ansehen, wo das allmähliche Umsichgreifen der Veränderungen, sowohl in den episkleralen und subconjunctivalen Theilen des Bulbus nach rorn und in die Hornhaut, 
Weiterer Beitrag zur pathologischen Anatomie der Skleritis. 559

als auch auf das Innere des Auges unter dem Bilde einer mächtigen hyperplastischen Chorioiditis mit secundären Glaskörperveränderungen, Netzhautablösung u. s. w. schliesslich zur Erblindung führte.

Die häufige Doppelseitigkeit des Processes, das bohe Alter der Patienten, die gleichmässige Ausbreitung der Veränderungen über den ganzen vorderen Bulbusabschnitt, die Complication mit schweren intraocularen Veränderungen, der chronische und meistens maligne Verlauf, die später auftretende schmutzig bräunlichröthliche Verfürbung der erkrankten Parthieen mit zum Theil sulziger homogener Verdickung der Conjunctiva bulbi und tiefer parenchymatöser Trübung der Cornea, scheinen mir die Züge im Krankheitsbilde zu sein, die uns wohl berechtigen, dasselbe im Sinne der Mittheilungen aus der Fuchs'schen Klinik als etwas Besonderes hinzustellen, doch ist es dem Symptomcomplex einer primären Skleritis und Episkleritis zuzurechnen, wie ich glaube.

\section{Literaturverzeichniss.}

1) Alt, Compendium der normalen und patholog. Histologie des Auges. 1879.

2) Brailey, W. A., "Peculiar solid thickening of the ocular conjunctiva in a child, apparently tubercular". Transact. of the united. kingd. 1889. Vol. IX.

3) Baumgarten, „Ophthalmolog. histol. Mittheilungen“. Ein Fall von sklerosirender Keratitis v. Graefe's Arch. f. Ophthalm. XXII. 2. S. 185.1876.

4) Coppez, Henri, Tumeur orbitaire avec envahissement secondaire de la selérotique et de la comée. Areh. d'ophthalm. XV. p. 544.1895.

5) Donald Gun n, 1. Syphilitic (?) subconjunctival infiltration. p. (i8. 2. Syphilitic (?) infiltration of conjunctiva. p. 68-71. 3. Syphilitic (?) infiltration of ocular. conjunctiva. p. $71-72$. Transactions of the ophthalm. Soc. of the united kingdom. 1894. V. XIV.

6) Friedland, Fr., Zur pathologischen Anatomie des Skleritis. y. Graefe's Arch. f. Ophthalm. XLVIII. 2. S. 283.1899.

7) Gayet, Sur les tumeurs symétriques des deux orbites et leur caractere symptomatique. Arch. d'ophthalm. p. 15. 1886.

8) Gayet, Deux tumeurs symétriques des globes oculaires. Arch. d'ophthalm. 1888. p 18 .

9) Kostenitsch, Ueber einen Fall von Skleritis. Pathol.-anatom. Untersuch. Arch. f. Augenheilk. XXVIII. S. 27. 1894. 
560 W. Uhthoff, Weiterer Beitrag zur pathol. Anatomie der Skleritis.

10) Noyes, H., Transact. of the Americ. ophthalm. Society 34-42. 1873. Ref. Nagel's Jahresber. f. Ophthalm. 1873. S. 279.

11) Pilz, Prager Vierteljahrrschr. Bd. XXXVI. S. 166-200. 1852.

12) Schirmer, 0., Zur pathol. Anatomie der Skleritis und Episkleritis. v. Graefe'sArch. f. Ophthalm. XLI. 4. 1895.

13) Schlodtmann, W., Ueber sulzige Infiltration der Conjunctiva und Sklera. v. Graefe's Arch. f. Ophthalm. XLIII. 1. 1897.

14) Uhthoff, W. Beiträge zur pathol. Anatomie des Auges. T. Skleritis und Episkleritis. v. Graefe's Areh. f. Ophthalm. XXIX. 3. 1883.

15) Uhthoff, W., Zur pathol. Anatomie der Skleritis. Bericht üb. d. Versammlung deutscher Naturforscher und Aerzte zu Frankfurt a. M. 1896. II. Th. 2. Hälfte. S. 326.

\section{Erklärung der Abbildungen auf Tafel XIII-XV,} Figur $1-5$.

Fig. 1. Horizontaldurchschnitt des linken enucleirten Auges, der die Ausdehnung und Intensität des pathologischen Processes erkennen lässt.

Fig. 2. Durchschnitt durch eine skleritische Parthie im vorderen Bulbusabschnitt, wo die Sklera ganz durchsetzt ist von dem Entzündungsprocess.

Fi g. 3. Einzelne Knötchen und die Entwicklung derselben aus Endothelwucherung in einem Gefässquerschnitt.

Fig. 4. Theil eines solchen Knötchens bei stärkerer Vergrösserung. Das Centrum endotheliale Zellen enthaltend mit einer ringförmigen Infiltrationszone einkerniger Leukocyten. Nenbildung kleiner Gefassse, welche sowohl die Peripherie als das Centrum durchziehen.

Fig. 5. Durchschnitt der entzündlich veränderten Chorioidea, mit starken Veränderungen der Gefässwandungen. 


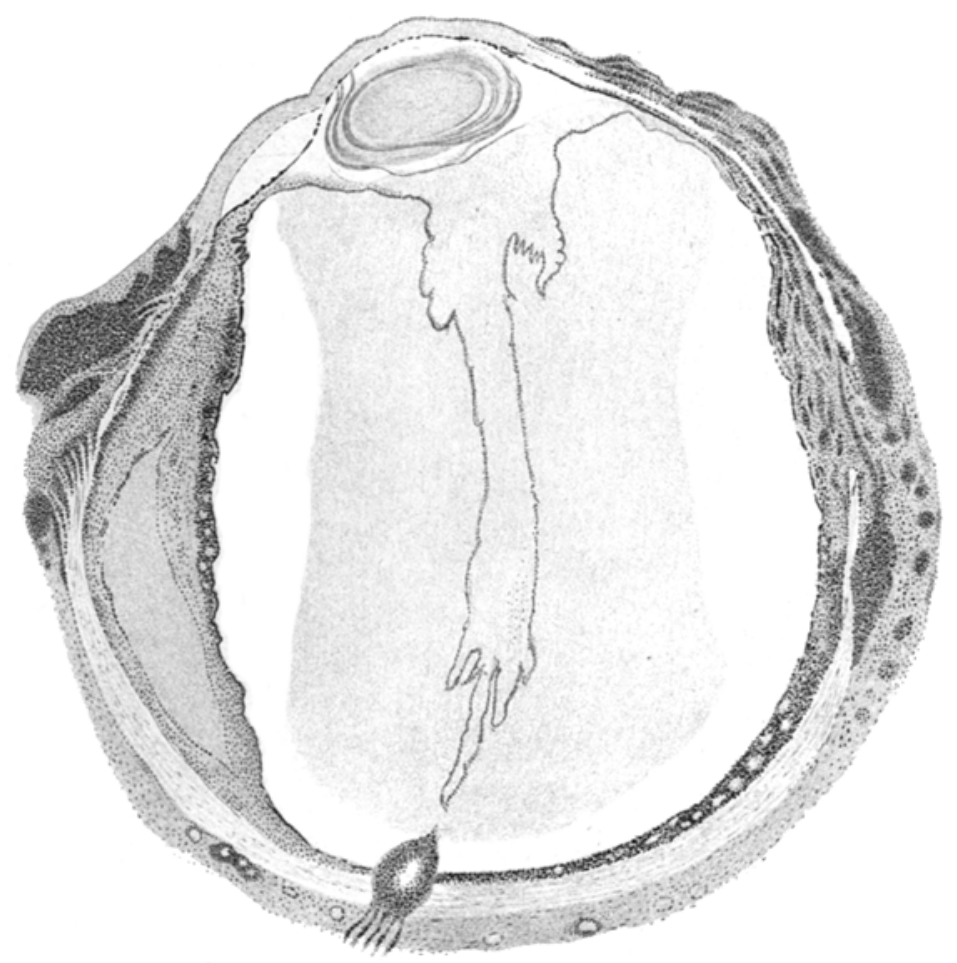




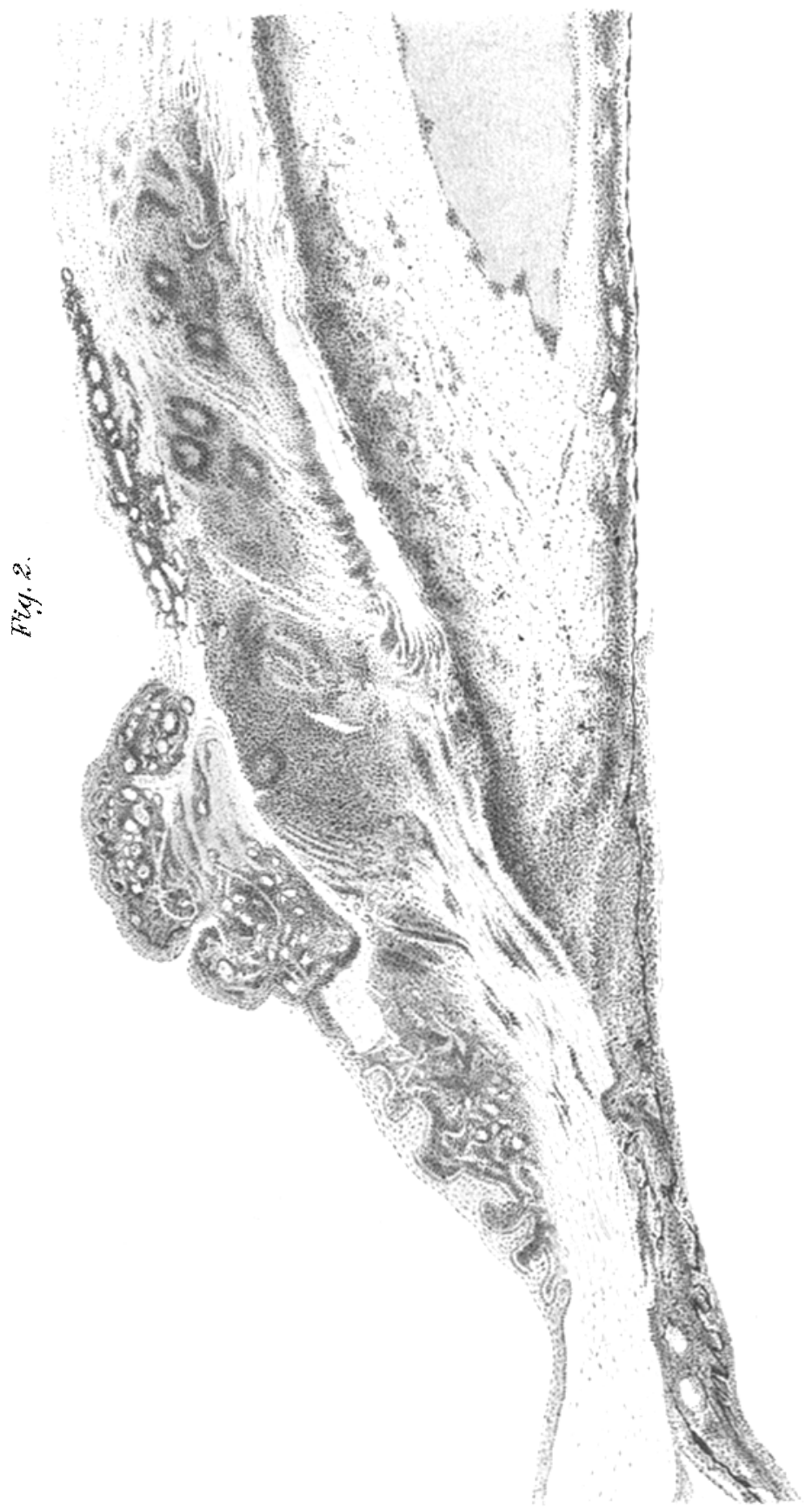

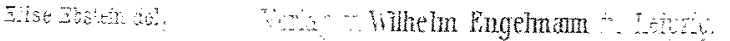

Hons 


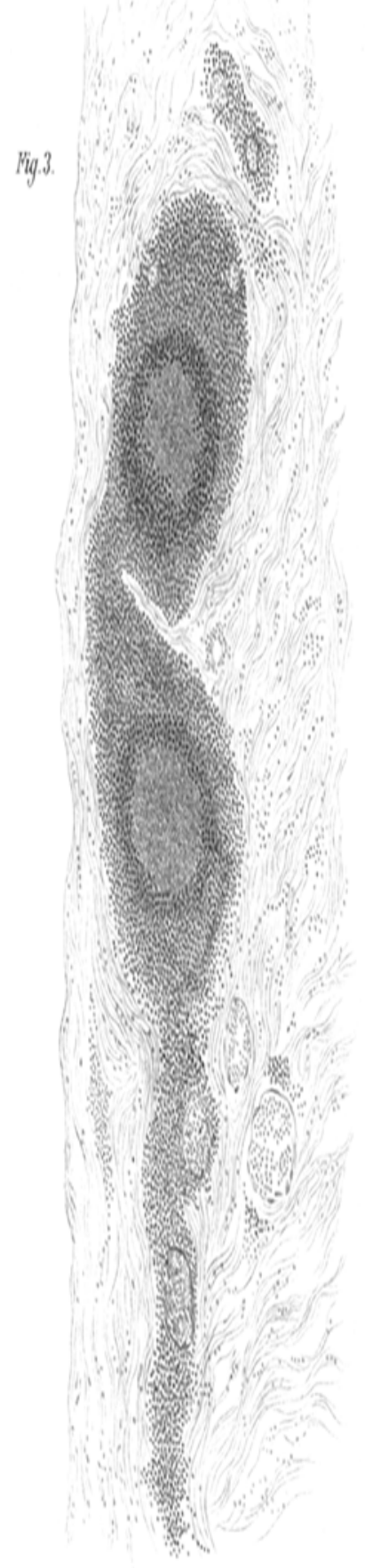

Fig. 4 .
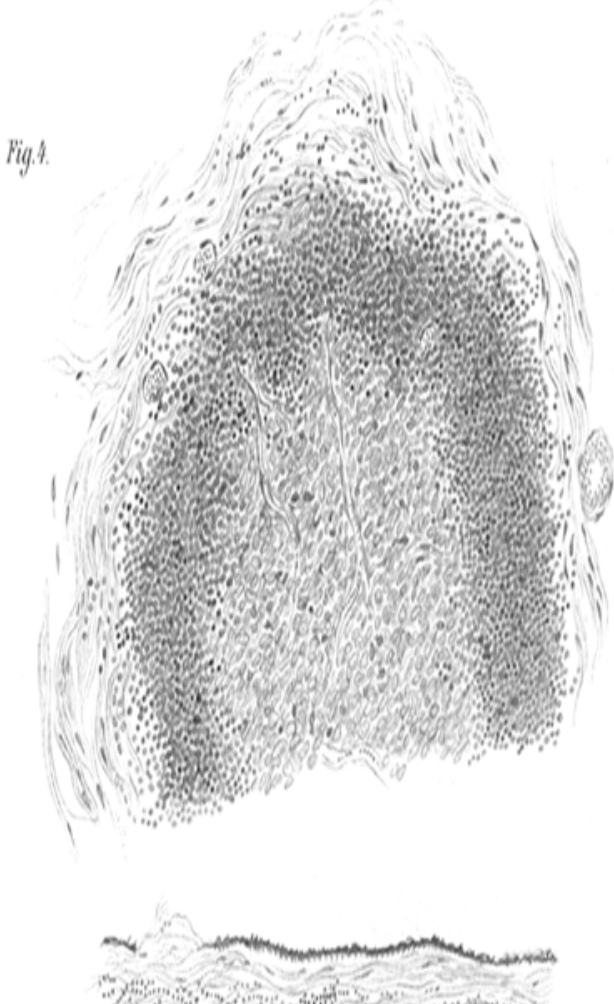

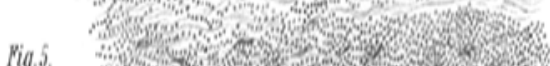

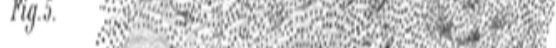
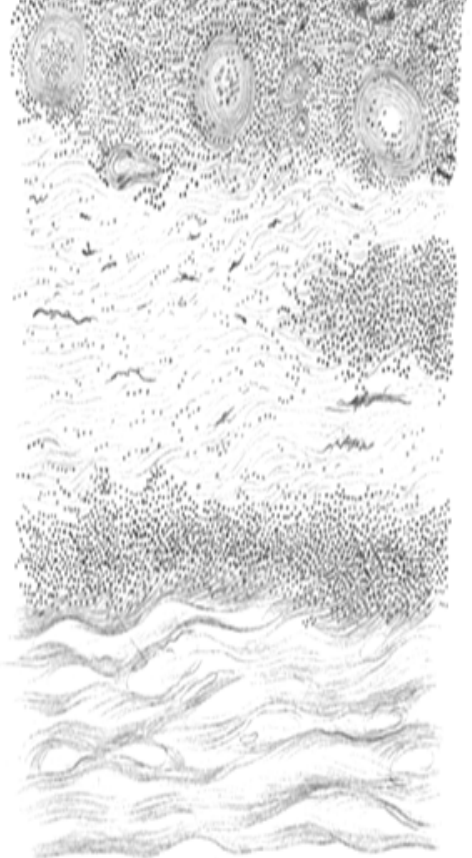